\title{
An Energy Balancing LEACH Algorithm for Wireless Sensor Network ZHANG Xian $\mathrm{Li}^{1, \mathrm{a}}$, LI Qian ${ }^{1, \mathrm{~b}}, \mathrm{FU} \mathrm{Yu}^{1, \mathrm{c}}$ and *QIAN Zhi Hong ${ }^{1, \mathrm{~d}}$ \\ ${ }^{1}$ College of Communication Engineering, Jilin University, Changchun 130012, China

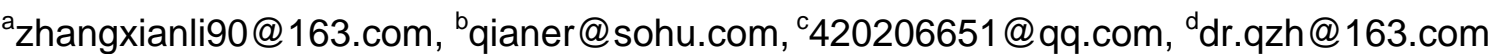

Keywords: wireless sensor network; LEACH protocol; the optimal cluster head; energy consumption

\begin{abstract}
As a typical hierarchical routing algorithm in wireless sensor network, LEACH protocol can reduce the routing overhead greatly. And it makes the network load balance relatively and have a good scalability. But there are still many deficiencies, such as the uneven clustering, unreasonable cluster head-selection, the single hop communication inter clusters and unsuitable for the large-scale network. Therefore, this paper proposes a new energy balancing routing algorithm, which determines the optimal number of cluster head based on the energy consumption of the network and selects the optimal cluster head based on network load balancing. The simulation results show that the algorithm can balance network load, prolong the network lifetime effectively.
\end{abstract}

\section{Introduction}

WSN (wireless sensor network) is a self organizing system. This system consists of sensor nodes, sink nodes and management nodes. And it is widely used in military, medical, environmental monitoring and so on. However, sensor nodes have the shortcomings such as energy limited storage capacity, low computing power and weak communication capabilities[1,2]; The network lifetime depends on the energy consumption of sensor nodes largely. Therefore, we can improve the efficiency of routing greatly in the network, reduce the routing overhead and the energy consumption of the nodes properly to reach the balance between energy consumption and communication[3,4]. It will greatly promote the development and application of wireless sensor networks.

The rest of the paper is organized as follows: section 2 introduced several famous papers about reducing energy consumption in LEACH protocol proposed before; section 3 described the network energy consumption model and the radio model in this paper; the fourth section analyzed the deficiency of the LEACH algorithm. In the fifth section we carefully described the proposed algorithm and discussed the performance of the improved algorithm by simulating the network lifetime. In the end, we gave the conclusion and our future work.

\section{Related work}

In order to make the WSN energy minimization, researchers have proposed many efficient routing protocols recently. Heinzelman first proposes the LEACH protocol [5]. It selects cluster head randomly which balances the network load and energy consumption. But it does not consider the residual energy of nodes; Then Heinzelman proposes LEACH-C routing algorithm, which is a centralized cluster head election algorithm. The base station selects the cluster head node according to the location and the residual energy information of nodes [6]. But it causes the instability of the network easily; Gao and Yoo propose PLEACH algorithm based on LEACH-C, which calculates the optimal number of cluster head nodes. It recognizes the sink node as the center of the network and divides region into equal size, and selects the cluster head according to the residual energy in various regions [7].Though it can make the cluster distribution more uniform, but it did not consider the influence of the number of clusters and cluster size[8,9] on the network balancing.

Based on the above discussion, this paper proposed an improved algorithm for LEACH routing based on optimal cluster head. The algorithm gets the relationship between the total energy consumption of nodes and the cluster head number through the analysis of the influence factors on 
the optimal number of clusters, and deduces the optimal cluster head ratio formula. And it gives an energy balancing clustering routing algorithm in wireless sensor network, which considers node residual energy and the distance between the member node and sink node in the cluster head election process to balance the node energy consumption and prolong the network lifetime.

\section{Network energy consumption model}

At the stage of selecting the cluster head in LEACH protocol, every node generate a random number between $[0,1]$. If the number is less than the threshold $T(n)$ (which is determined by the formula (1) ), the node will become a cluster head. $p$ is the percentage of cluster heads in all nodes. $r$ is the number of selection round. $\mathrm{G}$ is a collection of unselected nodes. Since then, the number can act as cluster heads is less. So the probability of other nodes becoming cluster heads will increase to ensure the number of cluster heads of each round. After $1 / p-1$ rounds, the probability of the node which is not selected as cluster node will be 1 . After $1 / p$ rounds, all nodes begin the random decision to act as cluster head or not. However, the cluster head selection methods did not consider the node residual energy and distance information.

$$
T(n)=\left\{\begin{array}{cc}
\frac{p}{1-p *\left(\operatorname{rmod} \frac{1}{p}\right)} & \text { if } n \in G \\
0 & \text { otherwise }
\end{array}\right.
$$

In this paper, we use the transmission system model which is proposed by reference[5] to calculate the energy consumption of the network. In the LEACH algorithm, the model have three conditions: all nodes are same and have limited energy in the network; The radio signals have same energy consumption in each direction; The position of base station is fixed. The model includes: the energy consumption of transceiver circuit, amplifier circuit and receiver circuit.

The node energy consumption of sending $l$ bits packet is:

$$
E_{T}(l, d)=E_{T \text { elec }}(l)+E_{T \text { amp }}(l, d)= \begin{cases}E_{\text {elec }} * l+E_{\text {elec }} * \varepsilon_{f s} * d^{2} & d \leq d_{0} \\ E_{\text {elec }} * l+E_{\text {elec }} * \varepsilon_{m p} * d^{4} & d>d_{0}\end{cases}
$$

Here $E_{\text {elec }}$ is energy consumption of transceiver or receiver processing 1 bit. And $d$ is transmission distance, $\varepsilon_{f s}$ and $\varepsilon_{m p}$ is energy consumption of transceiver of amplification circuit. $d_{0}$ is the transmission distance threshold value, and $d_{0}=\sqrt{\varepsilon_{f s} / \varepsilon_{m p}}$. If the transmission distance is less than the threshold value $d_{0}$, power loss model is to use free space model; And if the transmission distance is greater than or equal to the threshold value $d_{0}$, power loss model is to use multipath attenuation model.

Energy consumption of the sensor node receiveing $l$ bits message can be calculated as:

$$
E_{R}(l)=E_{R \text { elec }}(l)=E_{\text {elec }} * l
$$

Therefore, the energy consumption of $l$ bits data from the node $n_{i}$ transfered to $n_{j}$ can be expressed as:

$$
E_{i, j}(l)=E_{R}(l)+E_{T}(l, d)
$$

\section{Deficiency of the LEACH algorithm}

Although the LEACH algorithm has a better performance, but there are still many shortcomings:

(a) The selection of cluster heads is determined by the time only, and it do not have a relationship with the residual energy of nodes; cluster head selection frequently will lead to large amount of broadcast messages and the node energy cost.

(b) In the formation of cluster, all the non-cluster head nodes are involved, and the percentage of cluster heads in all nodes is the same all the way. So it will increase the complexity of the formation of cluster. 
(c) The cluster head nodes and the base station using direct communication to communicate. It may cause the more energy consumption of the sensor nodes which are far away from the base station.

(d) Using random cluster, the number of clusters generated in each round not the same and the cluster size is not the same too. The size and the number of clusters far away from the base station are bound to affect the balance of overall energy consumption of the network.

\section{The improved algorithm}

\section{Determining the optimal number of cluster head.}

With $n$ nodes uniformly distributed in a square area monitoring $A=M * M\left(m^{2}\right)$, assume that:

(a) The packet length is the same, and the retransmission and collision energy consumption is ignored ;

(b) the base station is in the center of the region, and distance from member nodes to the $\mathrm{CH}$ (Cluster head)and from $\mathrm{CH}$ to sink node satisfied $\mathrm{d} \leq d_{0}$.

Energy consumption of each cluster head node in each cycle mainly includes: energy of receiving the information of member nodes, energy of data fusion and sending the fused data to the base station. Assume the number of clusters is $\mathrm{k}$. Then, the average member nodes in each cluster is $N_{1}=n / k-1$. Energy consumption of each $\mathrm{CH}$ in one cycle is: $E_{C H}=\left(\frac{n}{k}-1\right) * E_{\text {elec }} * l+\frac{n}{k} *$ $E_{D A} * l+l * \varepsilon_{f S} * d_{B S}^{2} . d_{B S}$ is the average distance between $\mathrm{CH}$ and the base station. $d_{C H}$ is the average distance from member nodes to $\mathrm{CH}$. The operation process for $d_{B S}$ and $d_{C H}$ can be described as follows:

$$
\begin{aligned}
& d_{B S}=\iint \sqrt{x^{2}+y^{2}} * \rho(x, y) d A=\iint \sqrt{x^{2}+y^{2}} * \frac{1}{M^{2}} d x d y=0.383 M \\
& d_{C H}^{2}=\iint\left(x^{2}+y^{2}\right) * \rho(x, y) d x d y=\frac{M^{2}}{2 \pi k}
\end{aligned}
$$

Here, $\rho(x, y)$ is the density function of nodes. The energy consumption of transmission each bit data per cycle is $E_{\text {Cluster }}=E_{C H}+\frac{n}{k} * E_{\text {non } C H}$. So the total energy consumption of the network is :

$$
\begin{aligned}
& E_{\text {total }}=k * E_{\text {Cluster }} \\
& =l *\left[(2 n-k) * E_{\text {elec }}+n * E_{D A}+k * \varepsilon_{f S} * 0.146 M^{2}+(n-k) * \varepsilon_{f S} * \frac{M^{2}}{2 \pi k}\right]
\end{aligned}
$$

We can get the optimal number of cluster head:

$$
k_{\text {opt }}=\sqrt{\frac{n M^{2} * \varepsilon_{f s}}{2 \pi\left(0.146 M^{2} * \varepsilon_{f s}-E_{\text {elec }}\right)}}
$$

Furthermore, the optimal cluster ratio can be expressed as:

$$
P_{\text {opt }}=\frac{k_{\text {opt }}}{n}=\sqrt{\frac{M^{2} * \varepsilon_{f s}}{2 n \pi\left(0.146 M^{2} * \varepsilon_{f s}-E_{\text {elec }}\right)}}
$$

\section{ED-LEACH.}

After determining the optimal number of cluster head, Not only the number of clusters have an impact on the system energy consumption, But also the cluster head selection does. And it largely determines the survival time of clusters. So it is very important to select cluster head.

This paper selects the suitable cluster head node by considering the residual energy of the nodes in the cluster and the distance to the base station . The main idea is as follows: we should set up a limited conditions firstly, which is showed in formula (10).It include a distance visibility and a inspiration of residual energy. The cluster head node in the $N O . i$ cluster is $H_{i}$, and $T_{H_{i}}=$ $\operatorname{Min}(T(j)) . j$ is a node of NO.i cluster, $T(j)$ is a parameter of $j$ node about energy and distance. 


$$
T(j)=\left(\frac{d_{i}(j)}{d_{i \_a v e r}}\right)^{\alpha}+\left(\frac{E_{i \_a v e r}}{E_{\text {cur }}(j)}\right)^{\beta}
$$

Here, $d_{i}(j)$ is the distance to base station. $d_{i_{-} \text {aver }}$ is the average distance of all nodes to the base station distance. $E_{\text {cur }}(j)$ is the current energy of node $j, E_{i_{-} \text {aver }}$ is the average current residual energy of all nodes in the NO.i cluster. $\alpha$ is the weight of distance and $\beta$ is the weight of energy.

The simulation results and analysis.

For our experiments, we make $\mathrm{n}$ sensor nodes randomly and uniformly distributed in the area of $M * M\left(\mathrm{~m}^{2}\right)$ square. Assume the energy of base station is large enough and the communication radius can cover the entire network. We use the energy consumption model in the section 2, and calculate the energy consumption according to the formula (2). The model parameters is as follows:

$$
\varepsilon_{f s}=10 \mathrm{pJ} / \mathrm{bit} / \mathrm{m}^{2}, \varepsilon_{m p}=0.0013 \mathrm{pJ} / \mathrm{bit} / \mathrm{m}^{4}, E_{D A}=5 \mathrm{~nJ} / \mathrm{bit} / \mathrm{signal}, E_{\text {elec }}=50 \mathrm{~nJ} / \mathrm{bit} \text {. }
$$

Assume 100 nodes are randomly distributed in the monitoring area of $100 * 100\left(\mathrm{~m}^{2}\right)$, with the base station at location(50,50). In this paper, we use the lifetime of the network as the measure to analyze ED-LEACH algorithm, and the data is the average value of 50 times simulation experiment. Thus we obtained the contrast curves about the improved LEACH and the classic LEACH protocol on the network lifetime in the figure 1.

The simulation results show that the active node curve in ED-LEACH algorithm declines more flat than the classic LEACH protocol. Nodes in LEACH protocol begin to die after about 900 rounds and nodes in proposed algorithm begin to die after about 1100 rounds. This not only shows that the node energy consumption is less in each round of ED-LEACH algorithm,but also the number of alive nodes have a obvious growth over LEACH after runnig the same number of rounds. So, the improved algorithm can effectively prolong the network lifetime.

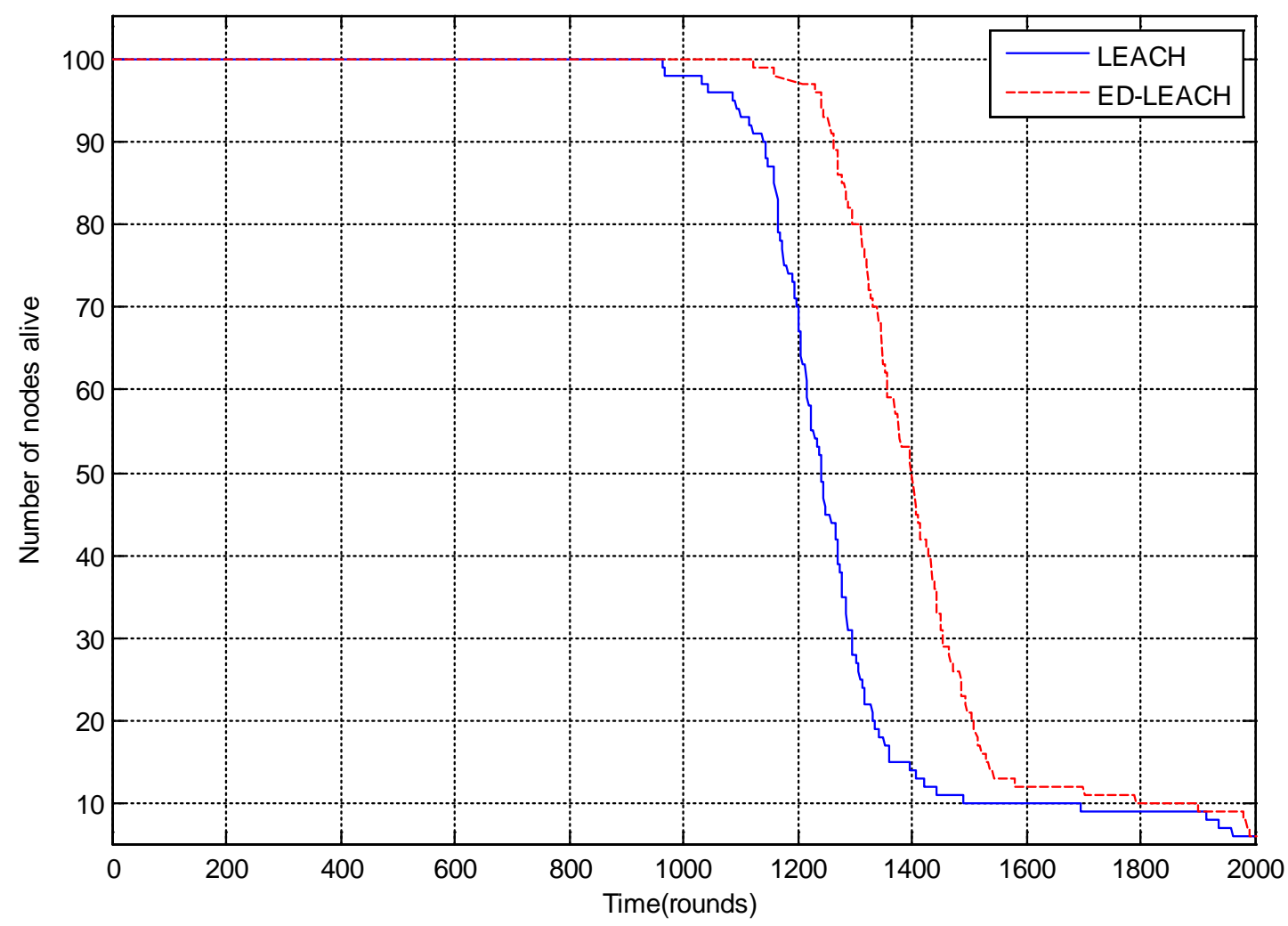

Figure 1. Simulation of network lifetime 


\section{Summary}

Through analyzing the factor which influence the number of cluster head, this paper determines the optimal number of cluster head. And it selects the cluster head considering the residual energy of node and distance from cluster head to the base station. The simulation results show that the improved algorithm not only prolong the network lifetime effectively, but also achieve the network load balancing. The network is developing toward large scale direction[10], but this paper did not take into account the applicability for large-scale wireless sensor networks of the LEACH protocol. So our future work is devoted into applying LEACH into large-scale wireless sensor networks.

\section{References}

[1] MCM T, Thein T. An Energy Efficient Cluster-Head Selection for Wireless Sensor Networks[C]. //Intelligent Systems, Modelling and Simulation (ISMS), 2010 International Conference on , Liverpool: IEEE, 2010:287 - 291.

[2] Shen B, Zhang S Y, Zhong Y P. Cluster-based routing protocols for wireless sensor networks[J]. Ruan Jian Xue Bao(Journal of Software), 2006, 17(7): 1588-1600.

[3] Salim M, Elsayed H, Ramly S. PR-LEACH: Approach for balancing energy dissipation of LEACH protocol for wireless sensor networks[C].//Radio Science Conference (NRSC), 2014 31st National , Cairo : IEEE, 2014 :252 - 259.

[4] Yassein. Bani M, Mistareehi. Improvement on the lifetime of the wsn using energy efficiency saving of leach protocol (New Improved LEACH)[J]. International Frequency Sensor Association, 2011, 130(7):142-154.

[5] Heinzelman W R. Energy-Efficient Communication Protocol for Wireless Microsensor Networks[C].//Proceedings of the 33rd Hawaii International Conference On System Sciences. Maui: IEEE Computer Society,2000:3005-3014.

[6] Heinzelman W R, Chandrakasan A P, Balakrishnan H. An application-specific protocol architecture for wireless microsensor networks[J]. Wireless Communications, IEEE Transactions on, 2002, 1(4): 660-670.

[7] Gou H, Yoo Y. An energy balancing LEACH algorithm for wireless sensor networks[C]//Information Technology: New Generations (ITNG), 2010 Seventh International Conference on. IEEE, 2010: 822-827.

[8] Khiati M, Djenouri D. BOD - LEACH: broadcasting over duty - cycled radio using LEACH clustering for delay/power efficient dissimilation in wireless sensor networks[J]. International Journal of Communication Systems, 2015, 28(2): 296-308.

[9] Bani Y M, Zeinab J, Hijazi, etal. New load balancing algorithm for LEACH protocol (F-VCH LEACH)[J]. International Frequency Sensor Association, 2012, 145(10):172-182.

[10] Qian Z H, Wang Y J. Internet of things-oriented wireless sensor networks review[J]. Dianzi Yu Xinxi Xuebao(Journal of Electronics and Information Technology), 2013, 35(1): 215-227. 